\title{
A novel approach to achieve breast symmetry in a single-stage procedure
}

\author{
Benedetto Longo, Rosaria Laporta, Marco Pagnoni, Fabio Santanelli di Pompeo \\ Department of Plastic Surgery, Sant' Andrea Hospital, School of Medicine and Psychology, Sapienza University of Rome, 00189 Rome, Italy. \\ Address for correspondence: Prof. Fabio Santanelli di Pompeo, Via di Grottarossa 1035-1039, 00189 Rome, Italy. \\ E-mail: fabio.santanelli@uniroma1.it
}

\begin{abstract}
Preservation of the skin envelope and the inframammary fold is the main factor in achieving breast symmetry in unilateral reconstruction. Skin sparing mastectomy (SSM) type-IV followed by immediate autologous reconstruction and contralateral symmetrization permits realizing this goal in large, ptotic breasted patients, and tumor superficially located in the inferior quadrants. If the tumor is superficially located in the superior or inferior quadrants with a previous lumpectomy or quadrantectomy scar in the superior quadrants, modified radical mastectomy and a staged procedure are recommended to avoid poor cosmetic results. Two patients who underwent immediate autologous reconstruction following SSM type-V with contralateral symmetrization in a one-stage procedure are presented.
\end{abstract}

Key words:

Autologous tissue reconstruction, breast symmetry, deep inferior epigastric perforator flap, single-stage breast reconstruction, wise-pattern mastectomy

\section{INTRODUCTION}

The re-creation of a natural-appearing breast mound while simultaneously achieving symmetry with the opposite breast represents a complex challenge during unilateral reconstruction. ${ }^{[1,2]}$

Skin-sparing mastectomy type-IV (SSM-IV), followed by immediate autologous reconstruction, and a simultaneous contralateral procedure is an ideal technique for large, ptotic-breasted patients with tumor located in the inferior quadrants (IIQQ). However, if the tumor is located in the superior quadrants (SSQQ) or IIQQ with a prior lumpectomy or quadrantectomy scar in the SSQQ, SSM-IV is contraindicated. In these cases, tumor resection interferes with wise-pattern (WP) skin flaps, and a modified radical mastectomy is instead recommended. As a result, a contralateral procedure to achieve symmetry becomes a complex, multifactorial decision, and a staged procedure

\begin{tabular}{|l|l|}
\hline \multicolumn{2}{|c|}{ Access this article online } \\
\hline Quick Response Code: & Website: \\
\hline & www.parjournal.net \\
\hline & \\
\hline
\end{tabular}

may be preferred to avoid a poor cosmetic result. This report presents two patients who underwent simultaneous contralateral mastopexy during unilateral SSM-V, followed by immediate deep inferior epigastric perforator (DIEP) flap reconstruction, as a complete single-stage procedure for upper quadrant skin and tumor resection.

\section{CASE REPORT}

\section{Case I}

A 56-year-old non-smoking woman was diagnosed with phyllodes tumor located deeply to IIQQ of her right breast. Medical history included repeated excisions of lumps and a superior-lateral quadrantectomy of the right breast. She had large (C bra-cup), ptotic (second-degree) breasts with a mid-clavicular to nipple distance of $28 \mathrm{~cm}$. She underwent a right SSM-V, axillary lymph-node dissection and immediate reconstruction with a $13 \mathrm{~cm} \times 21 \mathrm{~cm}$ de-epithelialized DIEP flap. Her nipple areola-complex (NAC) was grafted, and a simultaneous contralateral mastopexy was performed. The postoperative course was uneventful, and no complications were observed at the DIEP flap, SSM-V skin flaps, contralateral mastopexy, or and to the abdominal donor site. Breast symmetry of shape and size was achieved [Figures 1 and 2]. Neither surgical revision nor secondary procedures were required at her 20 months follow-up. 


\section{Case 2}

A 46-year-old non-smoking woman, with large (C bra-cup), ptotic (second-degree) breasts and mid-clavicular to nipple distance of $29 \mathrm{~cm}$, underwent a right SSM-V with axillary lymph-node dissection for a ductal carcinoma located in the superior-lateral quadrant, followed by immediate reconstruction with a $12 \mathrm{~cm} \times 18 \mathrm{~cm}$ de-epithelialized DIEP flap. Her NAC was grafted, and a contralateral mastopexy was performed in the same session. The postoperative course was uneventful. No complications were observed in the DIEP flap, SSM-V skin flaps, contralateral mastopexy or at the abdominal donor site. Breast symmetry of shape and size was achieved [Figures 3 and 4]. Neither surgical revision nor secondary procedures were required at follow-up of 16 months.

\section{DISCUSSION}

In unilateral breast cancer, the aesthetic quality of the reconstruction is also judged on the basis of symmetry of shape and size with the opposite breast. This often requires simple adjustments achieved by contralateral breast reduction, mastopexy or augmentation. Factors affecting the choice of surgical procedure for the contralateral side include the patient's anatomic breast characteristics, the surgeon's preferences, the patient's desires, mastectomy type and reconstructive procedure.

The ideal time to perform symmetrization remains controversial due to the increased operative time and risk of complications with immediate reconstruction. Some argue that it is easier to adjust the opposite breast once the reconstructed breast has reached a stable shape, volume,

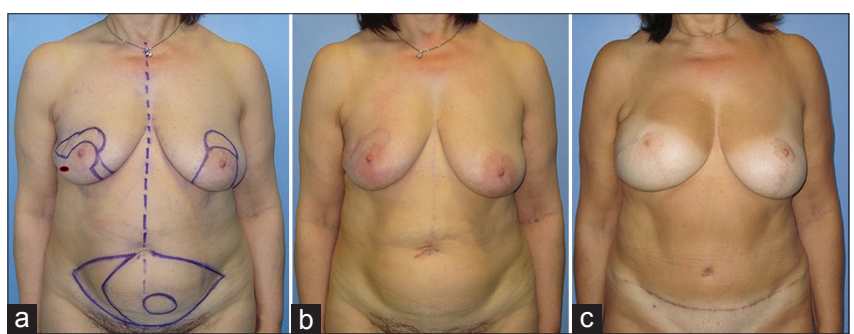

Figure 1: Case 1. A 56-year-old non-smoking woman with phyllodes tumour (black dot) located in inferior-lateral quadrant of the right breast and a previous quadrantectomy scar in the upper right pole. (a) Preoperative markings; (b) pre- and (c) postoperative frontal view

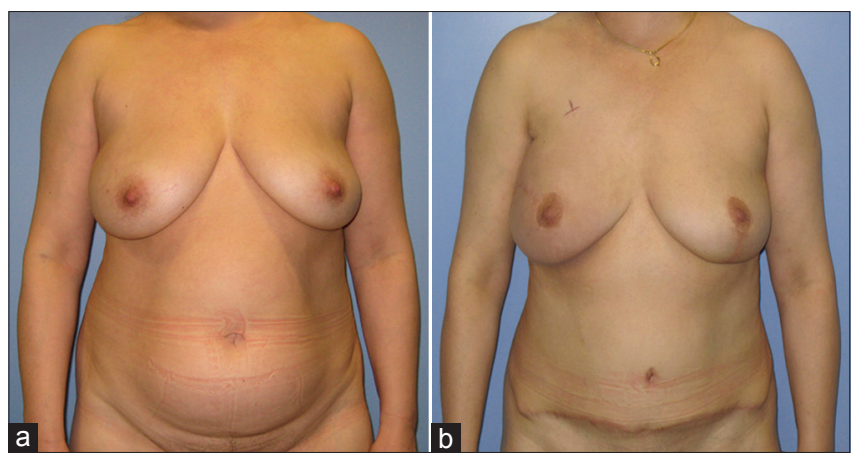

Figure 3: Case 2. A 46-year-old non-smoking woman with a ductal carcinoma located in superior-lateral quadrant of the right breast. (a) Pre- and (b) postoperative frontal view and position and only after completion of any adjuvant therapy in order to avoid potential disadvantages. ${ }^{[3,4]}$ In contrast, Stevenson and Goldstein ${ }^{[5]}$ observed that the combination of transverse rectus abdominus myocutaneous flap reconstruction and immediate contralateral symmetrization neither increased morbidity nor decreased aesthetic satisfaction. Losken et al. ${ }^{[6]}$ also confirmed superior aesthetic results with a simultaneous approach because the corrected opposite breast becomes the model for breast reconstruction rather than the other way around. In this context, the preservation of the skin envelope and inframammary fold is the key element to achieving an optimal shape and size with the opposite side during the initial surgery. SSM-IV, immediate autologous reconstruction and contralateral symmetrization represents an excellent single-stage procedure for large, ptotic-breasted patients with tumor located in IIQQ. Success of this procedure depends on WP application to both breasts that will lead to the same shape, projection and degree of ptosis since the preserved skin envelope is comparable between the two breasts. ${ }^{[7,8]}$ Moreover, it saves the patient a second surgical procedure under general anesthesia with less psychological and emotional distress, while lowering operating room costs and time on waiting lists.

The aim of this report was to illustrate how the same goal can be achieved in patients with large, ptotic breasts, but with tumor lying superficially in the SSQQ or deep to the

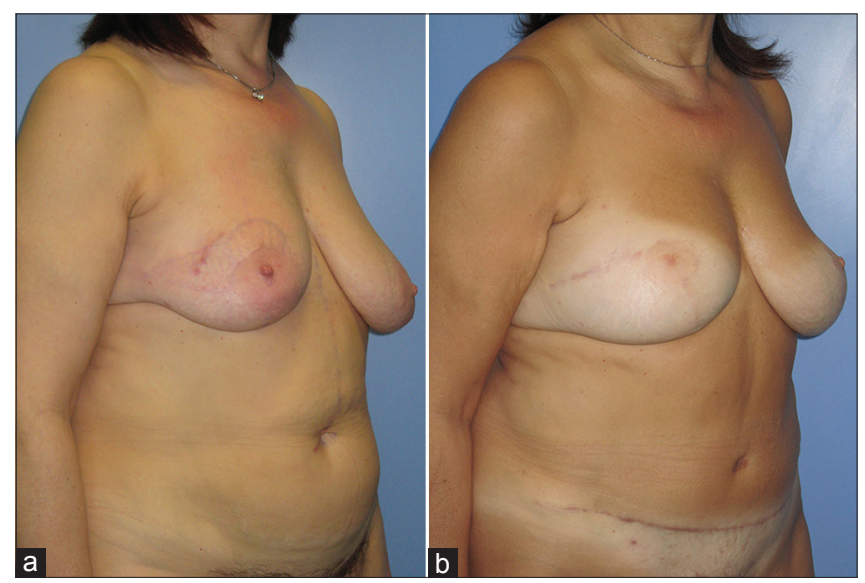

Figure 2: Case 1. (a) Pre- and (b) postoperative oblique view

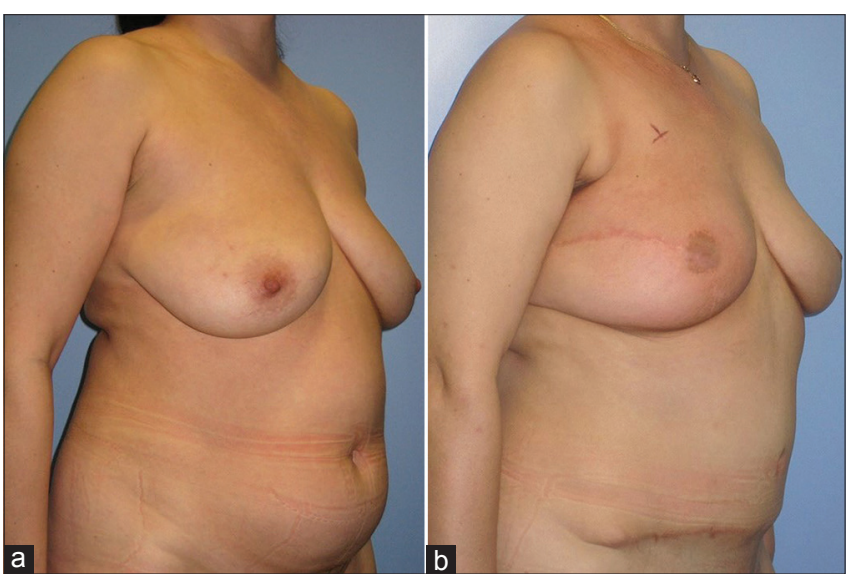

Figure 4: Case 2. (a) Pre- and (b) postoperative oblique view 


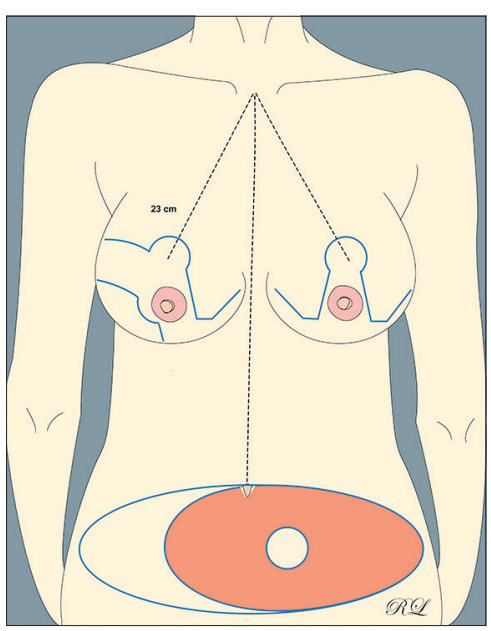

Figure 5: Modified wise-pattern for upper quadrant skin resection (skinsparing mastectomy type-V) was applied on the affected breast. Standard wise-pattern was used to perform mastopexy/reduction on the opposite side. Deep inferior epigastric perforator flap markings with a round skin paddle and the de-epithelialized area (pink color) of the flap. The round skin paddle can replace the nipple areola-complex (NAC) if intraoperative frozen sections are positive

IIQQ with a prior lumpectomy or quadrantectomy scar in the upper quadrants.

The reported procedure entails a modified WP-SSM for upper quadrant skin resection, as described by Santanelli et al., ${ }^{[9,10]}$ followed by immediate DIEP flap reconstruction and a contralateral symmetrization procedure. With the patient in standing position the median breast line was marked and the new nipple position was located at $23 \mathrm{~cm}$ from the sternal notch, then the WP was marked bilaterally. The general surgeon drew the skin area to be removed with breast parenchyma on the affected side and the plastic surgeon applied a modified WP to plan the SSM-V, while a "standard" WP was used to perform a mastopexy or breast reduction on the opposite side [Figure 5].

While the general surgeon performed the SSM-V with axillary lymph-node dissection, the plastic surgeons harvested the DIEP flap, tailoring it according to the final desired contralateral breast size. The flap was then transferred to the chest wall and revascularized by end-to-end anastomoses to the circumflex scapular vessels. The NAC was harvested and grafted if intraoperative frozen sections were negative [Figure 6].

There are many advantages to this novel approach. By preserving the skin envelope and infra-mammary fold on the affected side using a SSM-V, the WP can be applied to perform a simultaneous contralateral symmetrization, allowing both NACs to be placed at the same position. Furthermore, by preserving the skin envelope on the affected side a natural-appearing breast is achieved especially after autologous tissue reconstruction. Scarring is comparable to the SSM-IV with an additional equatorial scar located at the superior medial/ateral quadrant, which is less disfiguring when compared with a conventional mastectomy. Despite its surgical complexity, immediate DIEP flap reconstruction is the best chance for obtaining long-term symmetry because both breasts maintain natural ptosis and softness. ${ }^{[11,2]}$

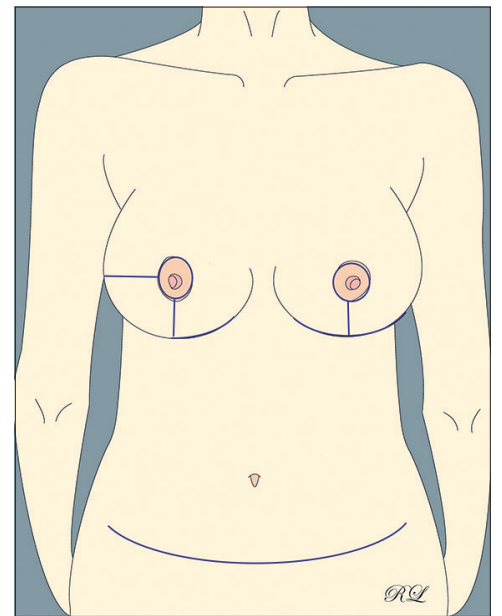

Figure 6: Scarring is comparable to the skin-sparing mastectomy type-IV with an additional equatorial scar located at the superior medial/lateral quadrant, which is less disfiguring when compared to a conventional mastectomy

\section{REFERENCES}

I. Kroll SS, Coffey JA Jr, Winn RJ, Schusterman MA. A comparison of factors affecting aesthetic outcomes of TRAM flap breast reconstructions. Plast Reconstr Surg 1995;96:860-4.

2. Blondeel PN, Hijjawi J, Depypere H, Roche N, Van Landuyt K. Shaping the breast in aesthetic and reconstructive breast surgery: an easy three-step principle. Plast Reconstr Surg 2009; 123:455-62.

3. Chang DW, Kroll SS, Dackiw A, Singletary SE, Robb GL. Reconstructive management of contralateral breast cancer in patients who previously underwent unilateral breast reconstruction. Plast Reconstr Surg 200 I; 108:352-8.

4. Labandter HP, Dowden RV. Surgical considerations in managing the remaining breast during postmastectomy breast reconstruction. Clin Plast Surg 1984; I I:365-8.

5. Stevenson TR, Goldstein JA. TRAM flap breast reconstruction and contralateral reduction or mastopexy. Plast Reconstr Surg 1993;92:228-33.

6. Losken A, Carlson GW, BostwickJ 3rd, Jones GE, Culbertson JH, Schoemann M. Trends in unilateral breast reconstruction and management of the contralateral breast: the Emory experience. Plast Reconstr Surg 2002; I 1 0:89-97.

7. Jahkola T, Asko-Seliavaara S, von Smitten K. Immediate breast reconstruction. Scand J Surg 2003;92:249-56.

8. Hudson DA, Skoll PJ. Single-stage, autologous breast restoration. Plast Reconstr Surg 200I; I08:I I63-7I.

9. Santanelli F, Paolini G, Campanale A, Longo B, Amanti C. Modified wisepattern reduction mammaplasty, a new tool for upper quadrantectomies: a preliminary report. Ann Surg Oncol 2009; 16:1 I 22-7.

10. Santanelli F, Paolini G, Campanale A, Longo B, Amanti C. The "Type V" skin-sparing mastectomy for upper quadrant skin resections. Ann Plast Surg 2010;65:135-9.

II. Granzow JW, Levine JL, Chiu ES, Allen RJ. Breast reconstruction with the deep inferior epigastric perforator flap: history and an update on current technique. J Plast Reconstr Aesthet Surg 2006;59:57I-9.

12. Blondeel PN. One hundred free DIEP flap breast reconstructions: a personal experience. Br J Plast Surg 1999;52:104-II.

How to cite this article: Longo $B$, Laporta $R$, Pagnoni $M$, di Pompeo FS. A novel approach to achieve breast symmetry in a single-stage procedure. Plast Aesthet Res 2015;2:76-8.

Source of Support: Nil, Conflict of Interest: I myself Fabio Santanelli di Pompeo have submitted for publication on Plastic and Aesthetic Research a manuscript entitled: "A novel approach to achieve breast symmetry in a single-stage procedure". I, hereby certify, that to the best of our knowledge no financial support or benefits have been received by me or any co-author, by any member of my (our) immediate family or any individual or entity with whom or with which I (we) have a significant relationship from any commercial source which is related directly or indirectly to the scientific work which is reported on in the article.

Received: 12-10-2014; Accepted: 03-11-2014 